Revue des patrimoines

\title{
De la protection à la valorisation du patrimoine dévasté : penser la valorisation des ruines pendant la guerre
}

\section{Emmanuelle Danchin}

\section{OpenEdition}

Journals

Édition électronique

URL : http://journals.openedition.org/insitu/10920

DOI : 10.4000/insitu. 10920

ISSN : 1630-7305

Éditeur

Ministère de la culture

Référence électronique

Emmanuelle Danchin, « De la protection à la valorisation du patrimoine dévasté : penser la valorisation des ruines pendant la guerre », In Situ [En ligne], 23 | 2014, mis en ligne le 12 mars 2014, consulté le 19 avril 2019. URL : http://journals.openedition.org/insitu/10920; DOI : 10.4000/insitu.10920

Ce document a été généré automatiquement le 19 avril 2019

\section{(c)}

In Situ Revues des patrimoines est mis à disposition selon les termes de la licence Creative Commons Attribution - Pas d'Utilisation Commerciale - Pas de Modification 4.0 International. 


\title{
De la protection à la valorisation du patrimoine dévasté : penser la valorisation des ruines pendant la guerre
}

\author{
Emmanuelle Danchin
}

Face à l'accumulation des ruines matérielles dans les zones de combats, les questions du dédommagement et de la valorisation de certains vestiges se posèrent vivement dès le début de la Première Guerre mondiale ${ }^{1}$. Les villes et les villages français du front subissaient en effet, depuis le mois d'août 1914, l'avancée et le recul des armées, les combats et les assauts répétés de l'artillerie. Les destructions matérielles qui en résultèrent attestaient de l'efficacité de l'emploi intensif du matériel militaire. La disparition du patrimoine architectural - c'est-à-dire de l'habitat et notamment des formes artistiques et religieuses - devint vite un élément de mobilisation des populations (fig. $n^{\circ} 1$ ) (fig. $n^{\circ} 2$ ). 
Figure 1

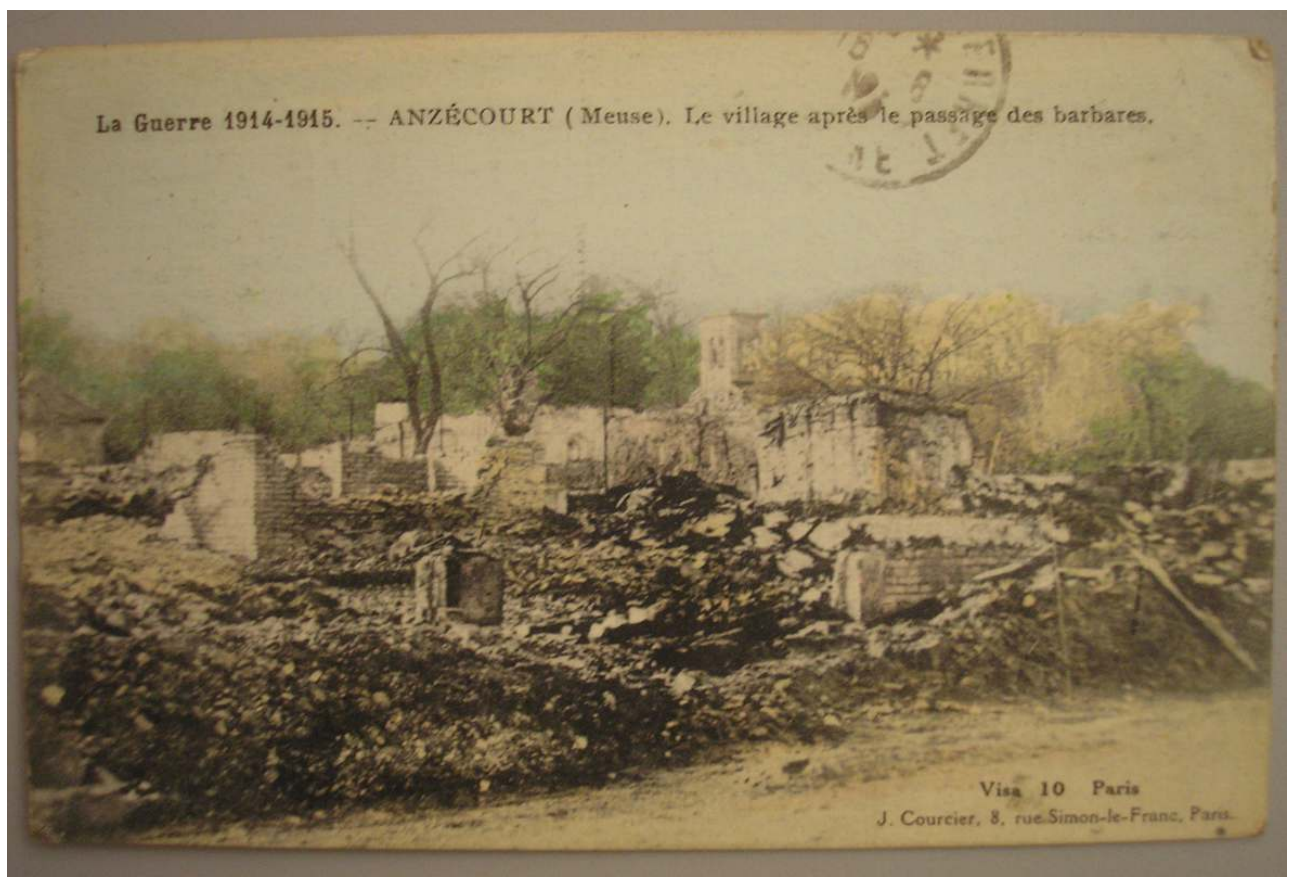

Anzécourt (Meuse), carte postale, boîte A.

(c) Historial de la Grande Guerre (Péronne)

Figure 2

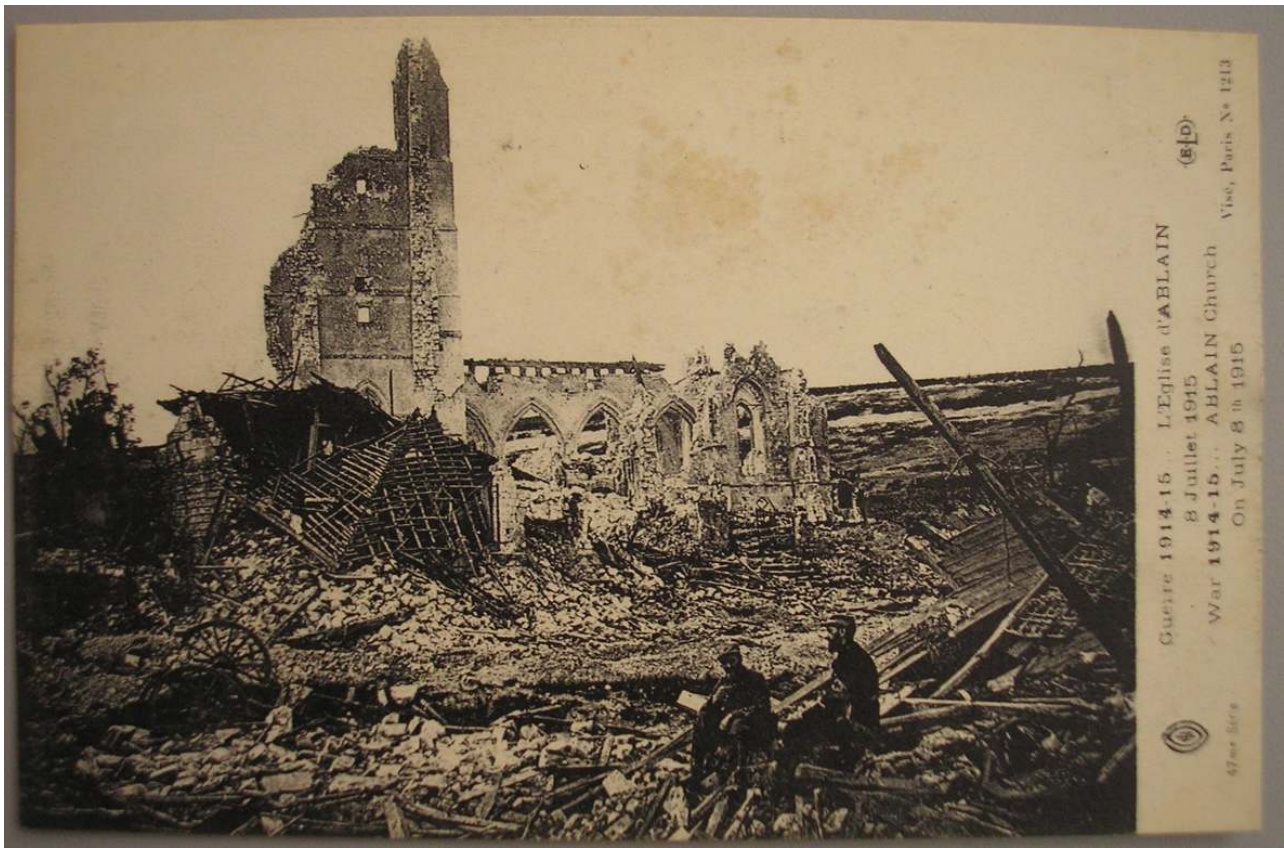

Église d'Ablain-Saint-Nazaire (Pas-de-Calais), carte postale, boîte A.

(c) Historial de la Grande Guerre (Péronne).

2 Immédiatement, la presse puis, dans les mois qui suivirent, les artistes, les intellectuels et les éditeurs dénoncèrent ces attaques et instrumentalisèrent les ruines. L'argumentaire, qui se développa jour après jour pour faire connaître ces dévastations perçues comme 
illégitimes, fut cautionné par les protestations du gouvernement français et d'artistes qui cherchèrent à alerter l'opinion publique internationale ${ }^{2}$. Ce furent avant tout les édifices civils, cultuels, et en particulier les monuments historiques, qui firent l'objet d'une levée de boucliers quand ils étaient touchés par les projectiles allemands ${ }^{3}$. Préserver les traces de la guerre, puis décider ou non de leur valorisation devinrent très vite des sujets de préoccupation tant pour le ministère de l'Instruction publique et des Beaux-Arts et le ministère de la Guerre que pour les acteurs du renouveau touristique, les associations et les élus locaux.

3 Cet article se propose d'éclairer ces mesures de protection et les débats naissant autour de la conservation des vestiges de guerre. Il s'attachera avant tout aux édifices et ensembles en ruines en prenant principalement appui sur les archives du ministère des Beaux-Arts conservées à la médiathèque de l'Architecture et du Patrimoine. Une attention plus particulière sera portée aux ruines, entendues ici comme toute construction ou ensemble bâti rendu inutilisable par suite de l'emploi de l'artillerie ou d'explosifs.

4 Il s'agira donc d'approcher un aspect de la valorisation du patrimoine en temps de guerre, en nous intéressant plus spécifiquement aux ensembles détruits.

\section{Les prémices d'une préservation des vestiges: protéger, valoriser, conserver les édifices dans la zone des armées}

\section{Premières mesures de protection}

5 Dès 1914, les premières mesures de protection physique des bâtiments ${ }^{4}$ furent prises contre l'artillerie. Elles furent isolées, s'appliquèrent à des cas locaux, déterminés, sans plan défini et concernèrent avant tout les monuments artistiques et religieux, plus que les groupements d'habitations. L'avance allemande ne pouvant être enrayée, les monuments restaient exposés à l'incendie et on craignait peu les faibles effets destructeurs de l'artillerie de campagne qui accompagnait les troupes en mouvement. L'urgence était le déplacement des œuvres susceptibles d'être pillées et incendiées, le retrait des échafaudages en bois posés avant-guerre en vue de restauration d'édifices religieux, comme à Reims sur la tour nord ${ }^{5}$, et, au besoin, l'évacuation des populations civiles.

6 La législation ne permettait pas à l'État d'intervenir sur le droit des communes, mais il pouvait s'occuper de la sauvegarde des œuvres dont il avait la responsabilité directe. Les grandes villes comme Lille, Nancy, Reims, Besançon et Épinal furent donc invitées à organiser l'évacuation de leurs propres musées. Toutes ne le firent pas et Reims usa, par exemple, de son droit de propriétaire pour refuser le transfert de ses collections.

7 Des mesures furent aussi prises pour la protection des grands édifices nationaux comme les cathédrales et les monuments historiques communaux. Dès le 15 septembre 1914, les préfets de l'Aisne, des Ardennes, de la Marne, de la Meurthe-et-Moselle, de la Meuse, du Nord, de l'Oise, du Pas-de-Calais, de la Seine-et-Marne, de la Somme et des Vosges furent invités à ouvrir des enquêtes pour déterminer les dommages subis sur les monuments classés de leur département ${ }^{6}$. 
8 Les architectes en chef des Monuments historiques effectuèrent des inspections et la délégation de la Commission des monuments historiques se rendit, par exemple, dès le 27 septembre à Reims, le 29 septembre à Soissons et le 31 octobre à Senlis, peu après les bombardements de ces villes, pour constater les dégâts et envisager les premières mesures de protection. Celles-ci se résumèrent à des déplacements temporaires d'œuvres et à la pose, comme à Reims ${ }^{7}$, de sacs de terre. À Amiens, qui n'avait pas souffert du passage allemand et des tirs, on amena de l'eau dans les combles de la cathédrale et on fit également un barrage de sacs pour parer à toute éventualité. Mais à Soissons, où des travées de la voûte haute de la nef s'étaient effondrées, les dégâts étaient impossibles à réparer du fait de la présence allemande à moins de $1800 \mathrm{~m}$ de portée de tir.

Concernant l'habitat civil, tant que le front ne fut pas stabilisé et les zones habitées à l'abri de nouveaux tirs, les mesures de première urgence s'en tinrent à des réparations provisoires permettant à ceux qui avaient fui temporairement, ou étaient restés sur place, de vivre dans leurs demeures.

Pour les maisons légèrement endommagées, et dans le cas d'une restauration partielle, les travaux de réfection étaient simples et se limitaient à des réparations de toitures, des obturations de trous dans les murs, des réfections de planchers, de plafonds, de cloisons intérieures crevées par le choc ou l'explosion d'obus, et par le remplacement ou la remise en état des portes et des fenêtres brisées.

Dans le cas de destructions matérielles trop importantes, la construction d'abris temporaires fut envisagée pour permettre aux habitants de regagner leurs logis en attendant les reconstructions définitives. Les habitants dont les maisons avaient été détruites purent ainsi se réinstaller, reprendre l'exploitation de leurs terres et de leurs fermes s'ils étaient agriculteurs, continuer leur activité s'ils étaient ouvriers, artisans et fonctionnaires, ou surveiller la reconstruction de leurs immeubles détruits s'ils étaient propriétaires.

\section{Penser la valorisation future des ruines dans la reconstruction}

Dès 1915, la question de la préservation des ruines de la guerre commença à être débattue. Elle fit l'objet de discussions et de propositions dans la presse, mais aussi au sein des administrations en charge du patrimoine. La volonté de garder en l'état les ruines, en tous cas celles des monuments artistiques, sembla à ce moment de la guerre faire l'unanimité.

En Belgique, Le Courrier de l'Armée évoqua, par exemple, le souhait d'architectes, d'hommes de lettres et du Sénat de s'occuper immédiatement de la reconstruction des cités détruites par la «Kultur teutonne». Dans la partie occupée du pays, le gouvernement allemand, qui avait envoyé des architectes chargés de faire des plans de reconstruction, se heurta en revanche aux architectes belges qui n'étaient d'accord ni sur la manière de reconstruire, ni sur le style à adopter. Les uns voulaient faire revivre ces communes dans leur cadre moyenâgeux, les autres au contraire faire du neuf, du confortable, du "contemporain ", tandis qu'une troisième école - la middelmate (voie médiane) - pensait qu'il fallait traduire dans l'architecture la saveur des choses anciennes, tout en utilisant les progrès actuels de l'art de bâtir. 

pas autant la communauté artistique et chacun s'accorda sur la nécessité de les préserver. Pierre Sambrée, dans Les Nouvelles, proposa dès 1915 de valoriser les ruines de Visé ${ }^{9}$ :

À notre avis il n'y a pas à hésiter, il ne faut pas toucher à Visé ; il faut l'entourer d'une ceinture protectrice; il faut que l'on veille sur ses ruines; il faut que Visé apprenne au monde la barbarie teutonne; il faut que Visé soit une leçon de tous les jours, où les Belges viendront chercher des aliments pour entretenir la haine sacrée et où les étrangers contempleront les bienfaits de la Kultur germanique.

Quant à Visé, la cité vivante, on la reconstruira, en aval, entre les ruines actuelles et ce qui reste du village de Mouland.

On ira à Visé comme on va à Pompéi, et l'on comparera la férocité des éléments à la fureur teutonique. Ce sera édifiant et instructif.

Visé ville martyre et ville-emblème, tu rappelleras à nos enfants un des crimes les plus honteux qui aient souillé l'humanité !

Et en même temps, tu apporteras une aide efficace à ceux qui, jadis t'habitèrent et qui s'en furent rebâtir plus loin une cité nouvelle.

Non, il ne faut pas toucher aux cendres de Visé! Ces cendres garderont le feu perpétuel de notre haine et de notre mépris ${ }^{10}$.

L'idée déjà bien affirmée de conserver un quartier, un édifice en ruine dans un but pédagogique, comme " une leçon de choses » pour les générations à venir, se retrouva ensuite dans nombre de prises de position et projets en Belgique comme en France; les mêmes questions se posèrent partout où les ruines étaient présentes.

En attendant que les projets aboutissent, des comptes rendus sur les destructions commises furent dressés dans les zones envahies pour le compte du sous-secrétariat d'État aux Beaux-Arts. Dès le mois de septembre 1914, des enquêtes ${ }^{11}$ furent ainsi menées sur les dégâts subis par les édifices classés du fait des opérations de guerre. Elles répondaient à trois buts essentiels : sauvegarder des témoignages nécessaires à l'histoire, évoquer devant les générations futures promptes à l'oubli la leçon des faits, et organiser pour un prochain avenir le pèlerinage universel aux champs de la guerre mondiale.

Pour l'heure, les préfets comme les architectes encore en fonction recueillaient des renseignements sur les édifices classés de leurs départements ${ }^{12}$. Après chaque visite, ils étaient tenus d'envoyer un rapport ${ }^{13}$, accompagné d'une fiche, comportant le nom du département, de l'édifice, la mention du classement quand celui-ci existait, mais aussi l'époque de construction, les particularités du bâtiment, les dégâts subis, les mesures de protection à prendre si nécessaire, et leurs observations sur les œuvres ${ }^{14}$.

Rien ne fut pour autant simple dans la zone de combats: dans le Pas-de-Calais, les services étaient désorganisés et Charles Genuys, inspecteur général des Monuments historiques, invité à se rendre à Arras peu de temps après les premiers bombardements ne put, par exemple, y pénétrer le 17 octobre $1914^{15}$. Dans le Nord, le préfet déclara quant à lui, dès le 23 octobre 1914, qu'il était impossible d'assurer la visite en raison de la désorganisation des services; l'ennemi occupant la région, l'affaire fut provisoirement suspendue. Dans l'Oise, les architectes des Monuments historiques ne purent circuler dans l'arrondissement de Senlis situé dans la zone des armées sans sauf-conduits délivrés par la préfecture de l'Oise ${ }^{16}$.

19 L'enlisement du conflit ne simplifia pas la tâche des préfets et des architectes, et l'architecte ordinaire des Monuments historiques Chenevier, dans une lettre au soussecrétaire d'État aux Beaux-Arts datée du 22 novembre 1915, se plaignit pour sa part du parcours du combattant à effectuer pour visiter les églises de la zone de Verdun ${ }^{17}$. Il y 
décrivit la difficulté d'obtenir pour commencer un laissez-passer des généraux commandants de la région fortifiée de Verdun - quinze jours d'attente - puis un permis pour circuler dans l'automobile du sous-préfet, qu'il n'obtint que sept jours plus tard, pour une validité d'une semaine seulement.

Ces rapports fourmillent cependant de précieux détails sur les atteintes aux monuments : l'état des couvertures, des planchers, des façades, des sculptures, des vitraux et du mobilier, et sur les mesures prises rapidement. La plupart d'entre eux, envoyés tout au long de la guerre, furent suivis d'effets puisqu'on s'appliqua à réparer, à protéger contre les bombardements et à classer les monuments qui ne l'étaient pas encore. Dans l'immédiat, les premiers travaux consistèrent à boucher les ouvertures, à restaurer les toitures ou du moins, à les couvrir, et à mettre en place des secours contre les incendies.

21 À partir de 1916, l'idée de collecter ce qui pouvait subsister des monuments " martyrisés ${ }^{18}$ " émergea aussi, afin qu'il ne soit pas davantage porté atteinte à ces œuvres et en prévision de la réédification des monuments détruits. Une section spéciale fut alors créée, composée d'hommes pouvant être recrutés parmi les auxiliaires, ainsi qu'un atelier de moulage attaché au ministère des Beaux-Arts.

Des fouilles furent entreprises parmi les ruines, pour retrouver et rassembler les fragments d'architecture et de sculpture des monuments historiques ayant un intérêt artistique. Une fois récoltés, ces fragments, considérés comme des documents précieux, furent photographiés, moulés, puis placés dans une salle spéciale du musée d'Architecture et de Sculpture. Un album de ces œuvres photographiées, figurant ce qu'étaient les monuments avant la guerre et après l'invasion, fut par la suite publié et diffusé en France. Une collection de fragments destinée à exalter le sentiment du beau chez les jeunes générations, à l'intention des écoles régionales des Beaux-Arts, se constitua par ailleurs.

\section{Que faire des monuments classés ou caractéristiques du patrimoine artistique français?}

Les architectes, les ingénieurs et les urbanistes émirent aussi des avis sur les destructions matérielles. Il faut néanmoins faire la distinction entre les communes, pour certaines détruites totalement, et les monuments pris isolément, principalement les monuments d'art et les édifices civils.

Pour les premières, le débat sur la conservation des ruines se posa peu. À l'exception de quelques lieux que l'on envisagea dès le départ de maintenir en l'état pour garder la mémoire de la guerre - comme Monchy-le-Preux dans le Pas-de-Calais - ne pas reconstruire les communes dévastées était inenvisageable et les débats portèrent avant tout sur la manière de les rebâtir. Pour les seconds, il en fut tout autrement, tout du moins pendant la guerre: préserver des édifices en ruines fut dès 1915 une évidence; restait à déterminer ces lieux et ces monuments.

La question de la préservation ou non des monuments en ruines souleva néanmoins des débats contradictoires, certains proposant de conserver en l'état les édifices ayant souffert de la guerre, d'autres, au contraire, de les réparer ou de les reconstruire dans leur état ante bellum.

Une décision unique ne pouvait de toute manière s'imposer du fait de l'ampleur des sites touchés, de la variété des atteintes à la pierre, qui soulevaient tantôt des difficultés 
techniques - travaux de consolidation ou de protection pouvant défigurer le bâtiment tantôt des difficultés pratiques - quel monument préserver plutôt qu'un autre?

Plusieurs solutions s'offrirent face aux édifices ruinés : soit les laisser en l'état afin de témoigner des actes de l'ennemi et conserver ainsi la mémoire de la souffrance passée ${ }^{19}$; soit laisser les traces de la dévastation s'effacer en avançant l'idée que, les ruines étant amenées à disparaître faute de tout pouvoir conserver, la reconstruction ne signifiait pas nécessairement l'oubli.

Dans le premier cas, la solution envisagée allait dans le sens des romantiques qui considéraient que le temps passé ne saurait être retrouvé, que la mort des édifices était annoncée, que les ruines permettaient la remémoration de la barbarie, mais aussi des positivistes pour qui le principe d'une restauration limitée, le maintien en l'état, était un signe de triomphe sur l'ennemi. Dans le second cas, la restitution du bâtiment était privilégiée pour mieux montrer la victoire sur le mal, en respectant pour les régionalistes leurs caractères distincts, ou en faisant table rase, pour les modernistes, de la tradition locale, en reconstruisant avec des procédés nouveaux : la fin des ruines comme symbole en quelque sorte de la nation renaissante.

Entre ces deux extrêmes, exista la possibilité de préserver quelques monuments éloquents en témoignage du vandalisme allemand. Ce fut finalement ce dernier courant qui l'emporta après la guerre.

Pour l'heure, la destruction des monuments était perçue comme une insulte pouvant entraîner deux réactions possibles: perpétuer à jamais le souvenir de l'injure en conservant les ruines noircies et les voûtes éventrées comme un réquisitoire éternel, ou restituer aux monuments leur splendeur d'antan. Dès 1915, le clivage opposa donc surtout les partisans de la conservation à ceux de la reconstruction.

31 Pour les partisans de la préservation, il valait mieux laisser les ruines en l'état, afin qu'elles demeurent comme témoignage de la «barbarie allemande». Les ruines étaient donc une preuve matérielle qui témoignait pour les générations futures, «une leçon sinistre et toujours répétée, une accusation vivante toujours dressée, un bras de pierre toujours tendu ${ }^{20} »$.

32 Le problème se posait néanmoins, non seulement du choix de ruines aux formes suffisamment expressives ou imposantes pour garder le plus longtemps possible leur aspect tragique ${ }^{21}$, mais aussi de leur devenir. Les architectes et urbanistes avaient en effet conscience que l'œuvre de destruction n'était pas achevée, que les masses disloquées continueraient à se dissocier, que les agents atmosphériques concourraient à ce travail et que la végétation parasite envahirait les ruines, transformant au bout de quelques années les nobles ruines en amas informes de décombres. Il serait donc impossible à terme, et tous le reconnaissaient d'avance, de conserver des ruines sans y accomplir un travail confortatif très délicat et fort coûteux.

Pour les partisans de la reconstruction, les ruines devaient être balayées et laisser place à de nouveaux bâtiments, preuves de la renaissance du pays. Ils envisageaient malgré tout la conservation de quelques témoignages du vandalisme allemand.

Les églises et les hôtels de ville indispensables à la vie des cités seraient rebâtis car il était impossible, si grandiose que fût la désolation comme à Arras, de laisser pareils amas de décombres au cœur des villes de demain. D'autres, comme les vestiges de Coucy-leChâteau, déjà en ruines avant la guerre et dont les Allemands avaient nivelé les tours à la dynamite, ne méritaient pas, pour des raisons plus financières que techniques, d'être 
remontés; mais le châtelain pourrait très bien reconstruire, tout en conservant, par exemple, quelques traces des mutilations sur les façades. Quant aux édifices dont l'utilisation ne s'imposait pas, ils pourraient garder leurs blessures.

À cette époque, la reconstruction et la conservation des ruines furent donc conjointement envisagées. La presse comme les publications relayèrent ces débats et en abordèrent différents aspects : ceux relatifs aux populations, et notamment à la modernisation de l'habitat; ceux relatifs à la restauration des monuments historiques, et notamment la place faite à l'architecture régionaliste. L'idée de conserver un marquage matérialisant la ligne de front pour rappeler aux générations futures le souvenir de la Grande Guerre et le danger d'un retour offensif de l'ennemi fut même soulevée. Les populations qui avaient subi l'invasion voulaient, quant à elles, renaître à la vie et non vivre au milieu des ruines. Elles proposèrent de rebâtir pour les vivants, parfois de conserver quelques débris - des éléments d'architecture ou de sculpture de monuments détruits - pour les déposer dans un musée municipal ou un « reliquaire ».

Les enquêtes menées, tout d'abord en 1914 sur l'état des édifices classés, puis en 1915 sur ceux non classés, permirent la constitution d'une première liste de monuments que l'on entendait bien conserver ${ }^{22}$ comme vestiges de guerre et valoriser dès l'arrêt des combats.

\section{Du désir de classement à l'abandon des projets}

En 1917, le critique d'art André Michel expliquait dans la Revue des Deux Mondes, à propos des choix qui après la guerre se poseraient sur la préservation des monuments, que "quand les dernières hordes auront disparu, quand nous reviendrons dans nos villages, ou sur l'emplacement de nos villages, rasés -, quand nous rentrerons dans nos villes violées et leurs sanctuaires éventrés, nous ne pourrons nous borner à nous asseoir en pleurant sur des ruines ${ }^{23} »$.

Bien qu'il fût encore trop tôt pour émettre des avis définitifs sur les projets de conservation de l'après-guerre, le débat était déjà bien engagé sur le devenir des ruines. Les propositions étaient nombreuses, comme celle qui envisageait de créer une voie sacrée reliant les monuments dévastés de France et de Belgique ${ }^{24}$, ou celle proposant de remonter certains clochers comme celui de Tracy-le-Val, dans l'Oise. André Michel était favorable, pour sa part, au maintien en l'état, à Reims, des parties ruinées de la cathédrale pour que celle-ci continuât de vivre ${ }^{25}$. Il fallait, pensait-t-il, laisser les statues mutilées et n'en refaire aucune. Il fallait laisser béantes les cicatrices, laisser saignantes les plaies faites par les «barbares », laisser la nef démantelée ${ }^{26}$.

On entendait par ailleurs compléter la liste des monuments historiques et même, en cas de reconstruction, garder la trace de la dévastation par la pose de plaques perpétuant le souvenir de l'événement ${ }^{27}$.

Les services chargés de l'évaluation, de la protection du patrimoine et de l'évacuation des objets d'art se structurèrent davantage. Les enquêtes sur les édifices détruits ou endommagés à la suite des opérations de guerre se poursuivirent. Elles s'avérèrent difficiles, car soit les zones inspectées étaient encore sur la ligne de feu, comme en Meurthe-et-Moselle et dans les Vosges ${ }^{28}$, soit les transports manquaient et la circulation était chaotique, comme dans l'Oise ${ }^{29}$. 


\section{Le service des vestiges et souvenirs de guerre}

41 La Commission des vestiges et souvenirs de guerre fut instituée en 1917 pour étudier les conditions de classement et de conservation des vestiges de guerre ${ }^{30}$. Composée de représentants de différentes administrations (Guerre, Beaux-Arts, Travaux publics, Blocus et Régions libérées), elle désigna deux délégués - l'un au ministère de la Guerre, l'autre aux Beaux-Arts - pour procéder à une première reconnaissance des organisations dans les régions accessibles et nouvellement libérées de l'Oise et de la Somme.

La première inspection eut lieu en juin 1917, dans une zone qui s'étendait de NouvronVingré, à l'ouest de Soissons, à Tilloloy, au sud-ouest de Roye. Une seconde mission explora en septembre la région d'Arras et de Péronne.

Suite à ces visites de reconnaissance, des rapports furent établis, incluant une liste des vestiges à conserver, pour leur intérêt éducatif et historique, examinée par la Commission des vestiges qui effectua alors un premier tri. Constamment réévaluée jusqu'aux années 1920, cette liste montre combien il fut difficile, dès cette époque, de suivre les propositions de 1915 de conservation en l'état des communes ou des édifices ruinés ${ }^{31}$.

Les raisons de ce revirement progressif sont exposées sans détours dans les projets de classement: le coût exorbitant de l'entretien des futurs vestiges et la prise en considération de l'aspect périssable des ruines matérielles, qui s'abîmaient déjà sous l'effet de la dégradation naturelle, furent principalement pointés du doigt. La commission décida de réduire le nombre de projets et de retenir plutôt les monuments isolés, faciles à conserver sans frais, et sans inconvénient pour la reprise de la vie économique. Peu à peu, l'argument financier ${ }^{32}$ relégua au second plan l'argument mémoriel.

Pour perpétuer le souvenir des édifices non retenus, une documentation photographique et graphique, aussi complète que possible, fut également rassemblée ${ }^{33}$. La Section photographique et cinématographique de l'armée (SPCA) assura les prises de vues ${ }^{34}$.

Le service chargé de reconnaître l'état des monuments classés et de préserver les œuvres d'art de la zone des armées était constitué de trois architectes. Un opérateur SPCA fut délégué auprès de chacun d'eux. Il était chargé de photographier les monuments historiques, mais aussi les moyens mis en œuvre pour les protéger. Il devait en outre développer sur place ses clichés ${ }^{35}$. Ces missions photographiques, pour le compte du soussecrétariat d'État aux Beaux-Arts, collectèrent également des documents pouvant servir ultérieurement le développement touristique.

En attendant la fin de la guerre, la protection des édifices retenus fut confiée à l'autorité militaire. Pour faciliter le classement des futurs monuments et objets, l'administration des Beaux-Arts prépara un projet de loi afin d'étendre les dispositions de la loi du 31 décembre 1913 sur la conservation des monuments historiques aux vestiges et souvenirs de guerre. Ce projet stipulait :

Article Ier.- Peuvent être classés dans les conditions prévues par la loi du 31 décembre 1913, et seront soumis au régime de cette loi les immeubles et les objets mobiliers dont la conservation présente un intérêt national au point de vue de l'histoire de la guerre.

Toutefois, par dérogation à l'article 16, les objets mobiliers appartenant à des particuliers peuvent être classés, à défaut du consentement du propriétaire, dans les conditions prévues à l'article 5 de ladite loi.

Article 2.- Le classement est prononcé après avis d'une Commission spéciale comprenant deux Conseillers d'État, deux représentants des ministères de 
l'Agriculture, des Beaux-Arts, des Finances, de la Guerre, de l'Intérieur et des Travaux publics.

Cette Commission sera également consultée par le Ministre des Beaux-Arts pour toutes décisions prises en exécution de la présente loi.

Article 3.- Le classement des immeubles et objets susvisés entraîne pour leurs propriétaires l'obligation de les laisser visiter. Le Ministre des Beaux-Arts statuera sur les conditions générales de la visite qui pourra être soumise à un droit d'entrée. L'organisation de la visite pourra être concédée au département ou à la commune avec les recettes et les charges qui en découlent (...)

Article 4.- Il est interdit à tout propriétaire, locataire, usufruitier, occupant ou détenteur à un titre quelconque d'immeubles ou d'objets atteints par les faits de guerre, de tirer profit de leur visite et de les signaler par des affiches ou écriteaux $(. . .)^{36}$. des vestiges de guerre et le développement d'un tourisme des champs de bataille ${ }^{39}$. L'idée de conserver des traces du conflit fit dans l'ensemble l'unanimité et les supports photographique et cinématographique apparurent, dans un premier temps, comme des techniques idéales d'enregistrement et de conservation du patrimoine dévasté, 
conservant le témoignage de ce qui n'était plus tout en entretenant le souvenir de la destruction. Ces techniques permettaient de les immortaliser afin que les détériorations naturelles liées aux conditions atmosphériques (pluie, gel) mais aussi aux atteintes humaines (bombardements des mêmes lieux par l'un ou l'autre camp, vol et récupération d'éléments) n'aggravent pas l'état des bâtiments. Elles rendaient possible en outre l'enregistrement de traces immédiatement exploitables pour la propagande et en vue de restaurations ultérieures. L'enregistrement photographique et cinématographique du patrimoine dévasté accompagna donc les mesures de protection et les débats sur la valorisation des vestiges en prévision de l'après-guerre tout au long de celle-ci.

En 1921, le nombre d'édifices réellement classés et surtout de vestiges en ruines conservés était faible. Si on se réfère à la liste des sites à classer dressée cette année-là par la Commission des vestiges, sur les 249 emplacements recensés, seuls trente-quatre concernent des édifices en ruines, soit 13,6\% de l'ensemble des vestiges. Parmi eux, seul Coucy-le-Château ${ }^{40}$ est alors en instance de classement. Vingt-deux de ces édifices sont rayés des listes entre 1921 et 1922, les autres laissés en attente.

Si l'on s'en tient maintenant à la liste des vestiges de guerre officiels arrêtée par le Parlement, elle ne comprend plus en 1921 que trente sites $^{41}$, dont quatre châteaux: Tilloloy, Thiepval, Plessier-le-Roye, Coucy, les forts de Verdun et les villages d'Adremont, de Montfaucon, de Cumières et de la côte de l'Oie (fig. $\mathbf{n}^{\circ} \mathbf{3}$ ).

Figure 3

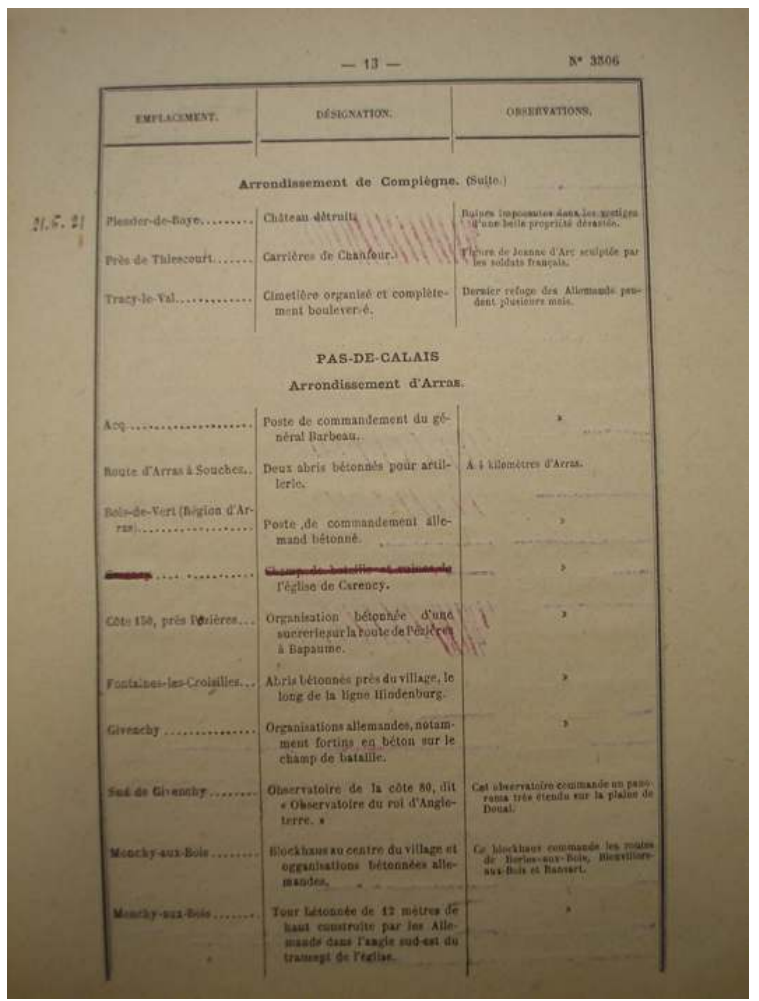

Extrait de l'annexe du rapport André Fribourg sur les vestiges et souvenirs de guerre en instance de classement, 1921, p. 13,80/3/6.

(c) Médiathèque de l'architecture et du patrimoine. 

mutilés rappelant les traces de la guerre et donc de la «barbarie allemande » a cédé la place à des impératifs purement pratiques, humains et financiers.

L'idée de conserver un marquage matérialisant la ligne de front pour rappeler aux générations futures le souvenir de la Grande Guerre et le danger d'un retour offensif de l'ennemi aboutit finalement en 1921 à la pose de bornes ${ }^{42}$ jalonnant la ligne de front telle qu'elle était le 18 juillet 1918, avant que l'envahisseur ne soit refoulée ${ }^{43} 119$ bornes commémoratives commencèrent à être posées à partir de 1921 (fig. $\left.\mathbf{n}^{\circ} 4\right)$. Le projet forma la Voie sacrée qui suivait la ligne extrême atteinte par l'envahisseur en 1918. Il fut achevé six ans plus tard.

\section{Figure 4}

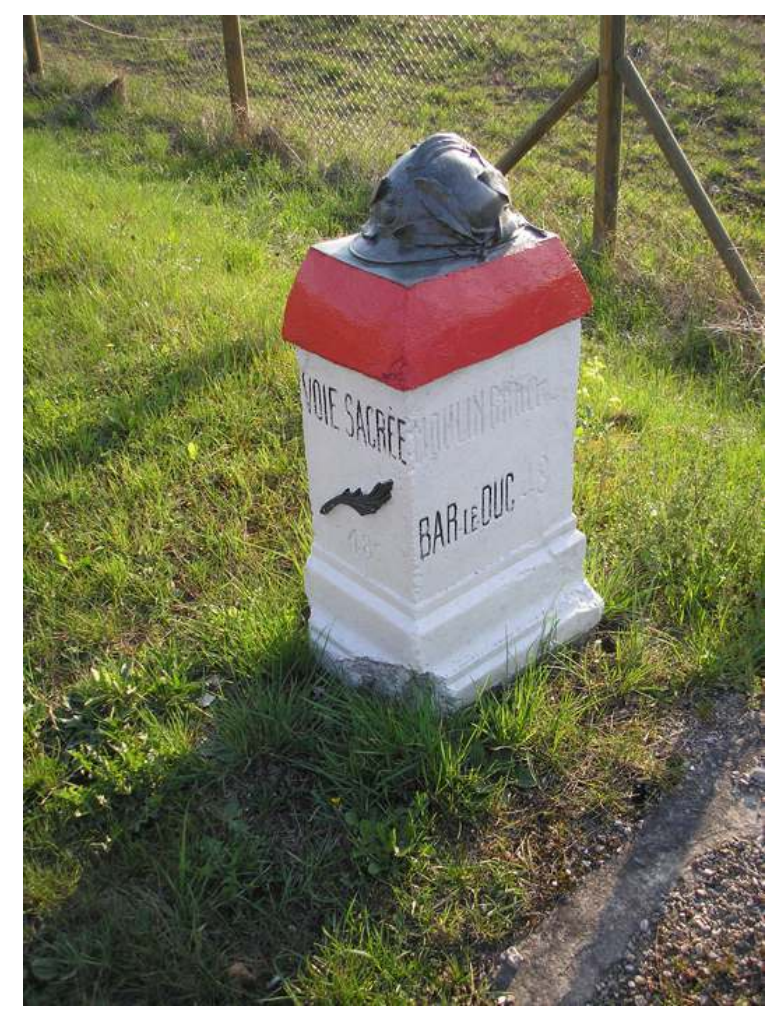

Verdun (Meuse), borne de la Voie sacrée à l'entrée de Verdun. Phot. Danchin, Emmanuelle. (c) E. Danchin.

\section{BIBLIOGRAPHIE}

Articles du même auteur

- « Voyage au pays des ruines : la nouvelle guerre pèlerins-touristes (1919-1921) », actes du colloque de Guise, 22-23 mars 2012 (à paraître). 
- « Photographier, inventorier et instrumentaliser le patrimoine architectural dévasté durant la Grande Guerre : regards sur les ruines françaises ». Dans NIVET, Philippe (dir.). Guerre et patrimoine artistique à l'époque contemporaine. Actes du colloque d'Amiens, 16-18 mars 2011. Amiens : Encrage édition, 2013, p. 151-163.

- «Les vues de dévastation prises par les soldats français, britanniques et allemands et mises en albums après la Première Guerre mondiale ». Dans DICKASON, Renée (dir.). Mémoires croisées autour des deux guerres mondiales. Actes du colloque de Caen, 27-28 mai 2010. Politeia. Paris : Mare \& Martin, 2012, p. 63-84.

- « Destruction du patrimoine et figure du soldat allemand dans les cartes postales de la Grande Guerre ». Amnis [en ligne : http://amnis.revues.org/1371].

- «Les ruines de la Grande Guerre vues du ciel ». Les chemins de la mémoire, n²12, février 2011, p. 13 [en ligne : http://fr.calameo.com/read/0003316273fbd25a3b50b].

- « Héroïsation des ruines et des combattants : la mise en place d'un tourisme de champ de bataille (1914-1921) ». Dans AUZAS, Vincent, JEWSIEWICKI, Bogumil (dir.). Traumatisme collectif pour patrimoine. Regards sur un mouvement transnational. Actes du colloque de Trois-Rivières du Québec, 7-8 mai 2007, PUL, 2008, p. 237-260.

- «Représenter la destruction pendant la Grande Guerre : les ruines consécutives aux premiers bombardements d'Arras d'octobre $1914 » .20 / 21$ (revue d'histoire des représentations), n 4 , hiver 2006-2007, p. 66-75.

\section{NOTES}

1. - Cet article s'inspire de notre travail de recherche de doctorat de $3^{\mathrm{e}}$ cycle sur les ruines de guerre et la nation française (1914-1921), soutenu en décembre 2012, et dont les résultats feront prochainement l'objet d'une publication. Ayant tout au long de ces années de thèse fait connaître régulièrement les avancées de celle-ci, c'est tout naturellement que nous avons accepté d'en livrer un aspect qui jusqu'à présent n'avait pas encore fait l'objet d'une valorisation particulière. 2. - Voir "Protestation au monde civilisé du gouvernement français », envoyée aux puissances signataires de la convention de La Haye et dénonçant l'achèvement des blessés, l'incendie systématique des villages traversés et le massacre des habitants, publiée notamment le 22 août 1914 dans le journal La Croix. Voir aussi la « Protestation du conseil des ministres » du 21 septembre 1914, et du conseil municipal de Paris qui s'associe immédiatement à la précédente, suite au bombardement de Reims.

3. - ROSENTHAL, Léon. Le martyre et la gloire de l'art français. Paris : Librairie Delagrave, 1916.

4. - Dossier « Dommages de guerre, mesures prises pour la sauvegarde des monuments et objets d'art ", 80/3/2, Médiathèque de l'architecture et du patrimoine (MAP) à Charenton-le-Pont.

5. - En 1913, un échafaudage en bois est érigé le long de la tour nord de la cathédrale de Reims en vue de restaurations. Lors du bombardement du 19 septembre 1914, son embrasement provoque un début d'incendie qui se propage ensuite à la charpente. Voir note $n^{\circ} 4$, Mesures prises pour la sauvegarde des monuments et objets d'art, novembre 1917, dossier « Dommages de guerre, mesures prises pour la sauvegarde... », op. cit.

6. - La France monumentale, texte dactylographié, dossier « Dommages de guerre, mesures prises pour la sauvegarde...", op. cit., et rapports et enquêtes sur les monuments historiques endommagés, dossier « Dommages de guerre. Enquête de 1915 sur les dégâts causés aux édifices classés et non classés », 80/3/3, MAP (Charenton-le-Pont).

7. - Dossier « Dommages de guerre, mesures prises pour la sauvegarde... », op. cit. 
8. - SAMBRÉE, Pierre. «La reconstruction de nos cités». Le Courrier de l'Armée, n 152 , 26 août 1915, p. 2.

9. - Visé, ville frontière belge des bords de la Meuse, fut une des premières localités au début du mois d'août 1914 à voir passer les troupes allemandes. Celles-ci comptaient franchir à cet endroit le fleuve pour gagner ensuite la place forte de Liège. Le $10^{\mathrm{e}}$ corps allemand occupa la ville et tenta de franchir à plusieurs reprises la Meuse par des ponts de bateaux. Le 10 août 1914, l'église de Visé fut incendiée et du 16 au 18 août la localité connut le pillage systématique et la destruction de près de 600 maisons et autres édifices (hôtel de ville, anciens couvents des Sépulcrines, des Récollets, maisons des Gildes).

10. - SAMBRÉE, Pierre. «La reconstruction de nos cités », op. cit.

11. - Voir notamment LÉON, Paul. La renaissance des ruines: maisons, monuments. Paris : Henri Laurens, 1918, p. 85.

12. - Minute de la lettre d'Albert Dalimier du 4 juillet 1916, dossier "Dommages de guerre. Demande de renseignements sur les édifices endommagés... ", op. cit.

13. - Dossier « Dommages de guerre. Demande de renseignements sur les édifices endommagés ... », op. cit.

14. - Ibid.

15. - Ibid.

16. - Lettre d'Albert Potdevin au sous-secrétaire d'État aux Beaux-Arts, 11 février 1915, dossier " Dommages de guerre. Demande de renseignements sur les édifices endommagés... », op. cit.

17. - Lettre de Chenevier, architecte ordinaire des Monuments historiques au sous-secrétaire d'État aux Beaux-Arts, 22 novembre 1915, dossier «Dommages de guerre. Demande de renseignements sur les édifices endommagés... ", op. cit.

18. - Proposition de Georges Legrand, sculpteur à Amiens, en vue de la réparation des dommages causés, et à l'effet de conserver tout ce qui a un intérêt d'art, de nos monuments détruits par le bombardement de l'ennemi, 21 février 1916, document dactylographié, dossier «Dommages de guerre. Demande de renseignements sur les édifices... ", op. cit.

19. - Cela fut envisagé par exemple pour l'église d'Ablain-Saint-Nazaire dans le Pas-de-Calais, ou la ville d'Ypres en Belgique dont le projet prévoyait de rebâtir une nouvelle cité à deux kilomètres du site d'origine.

20. - LAVEDAN, Henri. "La question de la cathédrale ». L'Illustration, n 3758, 6 mars 1915, p. 2 (p. 260 dans l'édition reliée).

21. - ROSENTHAL, Léon. Villes et villages français après la guerre. Paris : Payot, 1918, p. 269.

22. - Dossier "Dommages de guerre, mesures prises pour sauvegarder des monuments... », op. cit. 23. - MICHEL, André. «Dans les ruines de nos monuments historiques. Conservation ou restauration?». Revue des Deux Mondes, 15 novembre 1917, t. 42, p. 398-399.

24. - MICHEL, André, op. cit., p. 415.

25. - MICHEL, André, op. cit., p. 412.

26. - MICHEL, André, op. cit., p. 414-415.

27. - L'idée de plaques, se substituant aux éléments physiques ruinés non conservés, introduit un autre discours historique dans l'espace public. Voir à ce sujet, DUTOUR, Juliette. « Les plaques commémoratives ». Socio-anthropologie, $\mathrm{n}^{\circ} 19,2006$ [voir le site : http://socioanthropologie.revues.org/index603.html, consulté le 23 mai 2010].

28. - Lettre d'André Ventre au sous-secrétaire d'État aux Beaux-Arts, 26 juin 1916, dossier «État des travaux ou évacuation. Soissons 1916-1918 », 80/3/47, MAP (Charenton-le-Pont).

29. - Lettre d'Henri Bernard, architecte en chef des Monuments historiques au ministre de l'Instruction publique et des Beaux-Arts, 15 janvier 1918, dossier «État des travaux ou évacuation. Soissons 1916-1918 », 80/3/47, MAP (Charenton-le-Pont). 
30. - Note sur le classement et la conservation des Vestiges et Souvenirs de guerre, n.d., n.p., dossier « Vestiges de guerre. Législation. Projets de loi 1915-1923 », 80/3/36, MAP (Charenton-lePont).

31. - Voir la liste en annexe du rapport $n^{\circ} 3506$ du député André Fribourg à la Chambre, sur le classement des vestiges, 1921, dossier «Vestiges de guerre. Législation. Projets de Loi. 1915-1923», 80/3/6, MAP (Charenton-le-Pont).

32. - Rapport dactylographié du député André Fribourg à la Chambre, $n^{\circ} 3506$, op. cit., p. 3.

33. - Note sur le classement et la conservation des Vestiges et Souvenirs de guerre, op. cit.

34. - Voir à ce propos DANCHIN, Emmanuelle. «Photographier, inventorier et instrumentaliser le patrimoine architectural dévasté durant la Grande Guerre : regards sur les ruines françaises ». Dans NIVET, Philippe (dir.). Guerre et patrimoine artistique à l'époque contemporaine. Actes du colloque d'Amiens, 16-18 mars 2011. Amiens: Encrage édition, 2013, p.151-163. Sur le recrutement des opérateurs du Service photographique, se reporter à GUILLOT, Hélène. «Le métier de photographe durant la Grande Guerre ». Revue historique des armées, $\mathrm{n}^{\circ}$ 258, 2010, p. 87-102.

35. - Rapport sur la création et le fonctionnement, les résultats, de la Section cinématographique de l'Armée, 10 octobre 1917, dossier 1, boîte 2, Fonds archives du service photographique (1851-1999), p. 96.

36. - Projet de loi sur les souvenirs de guerre, novembre 1917, dossier « Vestiges de guerre. Législation. Projets de Loi. 1915-1923 », 80/3/6, MAP (Charenton-le-Pont).

37. - Le décret du 10 novembre 1920 transforme la Commission des vestiges et souvenirs de guerre, créée en 1917 pour se prononcer sur le classement définitif des vestiges retenus, en $4^{\mathrm{e}}$ section de la Commission des Monuments historiques.

38. - L'idée est formulée par le député Claude Cochin. Voir pour cela le Rapport dactylographié supplémentaire de Louis Marin à la Chambre des députés $\mathrm{n}^{\circ} 5896$, dossier «Travaux de protection exécutés en 1919 », 80/3/3, MAP (Charenton-le-Pont).

39. - Nous renvoyons à ce propos à notre article « Voyage aux pays des ruines : la nouvelle guerre pèlerins-touristes (1919-1921) », actes du colloque de Guise, 22-23 mars 2012 (à paraître).

40. - L'édifice est déjà sur la liste des immeubles classés au titre des Monuments historiques avant la loi du 31 décembre 1913 comme le précise le Jo du 18 avril 1914, p. 3576. Une demande de classement est tout de même faite après la guerre pour le château, l'église et les portes de Laon et de Soissons; la municipalité s'opposa au classement de l'intégralité du village. Voir dossier "Coucy-le-Château 02-Aisne », ministère de la Culture et de la Communication (Paris).

41. - Il s'agit soit de villages (Berry-au-Bac et Bellau dans l'Aisne, Moronvilliers dans la Marne, l'église et le village de Montfaucon, d'Avocourt, de Cumières-le-Mort-Homme, de Fleury-devantDouaumont dans la Meuse), soit de quartiers (le quartier entre la place de la Madeleine et la rue Mazet à Verdun dans la Meuse), soit de châteaux (Coucy, Soupir dans l'Aisne, Hattonchâtel, Grivesnes, Tilloloy, Coyencourt, Thipeval dans la Somme), soit de forts (la Malmaison dans l'Aisne, la Pompelle dans la Marne, Troyon en Meurthe-et-Moselle), soit de demeures (la Ferme de Bonne Maison dans l'Aisne, La Maisonnette, la propriété Dénecourt dans la Somme), soit d'églises (Saint-Hilaire dans la Marne, Miraumont dans la Somme, Richebourg dans le Pas-deCalais), soit d'établissements industriels (la sucrerie de Vic-en-Artois). Les autres sites sont des observatoires, des bas-reliefs, des entonnoirs de mines, etc.

42. - Ces bornes de $135 \mathrm{~cm}$ de haut, réalisées sur le modèle du sculpteur Moreau-Vauthier, rendaient hommage aux soldats qui avaient combattu pendant quatre ans. Exécutées en granit rouge d'Alsace, elles étaient coiffées d'un casque Adrian avec l'inscription: «ici fut repoussé l'envahisseur ». Elles pouvaient être acquises par souscription publique, par tous les pays dont les armées avaient combattu sur le sol français.

43. - H. D. [Henri DEFERT]. «Bornes commémoratives». Revue du Touring-club, $\mathrm{n}^{\circ} 328$, novembre 1921, p. 342. La pose des bornes commencée en 1921 est presque achevée en 1930. 


\section{RÉSUMÉS}

Dès 1914, le ministère de l'Instruction publique et des Beaux-Arts envisage les premières mesures de protection pour les édifices situés dans la zone des combats. Peu à peu émerge l'idée d'une préservation et d'une valorisation du patrimoine ruiné pour l'après-guerre. À partir de 1917, alors qu'est instituée la Commission des vestiges et souvenirs de guerre chargée d'étudier les conditions de classement et de conservation de ces vestiges, un revirement progressif s'opère néanmoins dans les projets. En 1921, il ne reste guère qu'une trentaine de projets de classement. Le souhait claironné pendant le conflit de préserver pour les générations à venir des monuments mutilés rappelant les traces de la guerre, et donc la «barbarie allemande ", a cédé la place à des impératifs purement pratiques, humains et financiers.

As early as 1914, the French Ministry of Public Education and Fine Arts took the first steps to protect the monuments located in the combat zones. The idea gradually took form of protecting and promoting the cultural heritage which had been devastated by the war. In 1917 the War Memory and Relics Commission (Commission des vestiges et souvenirs de guerre) was set up, entrusted with the task of reflecting on how to give conserve these relics by means of statutory protection.. However 1917 also marked the beginning of a change of attitudes towards such projects. By 1921, only about thirty projects for listing wartime monuments remained. The earlier desire to save for coming generations a selection of mutilated monuments bearing witness to the scars of the war and to 'German barbarism' gave way to more pragmatic, human and financial preoccupations.

\section{INDEX}

Mots-clés : Première Guerre mondiale, destruction, patrimoine, vestiges de guerre, ruines, classement

\section{AUTEUR}

\section{EMMANUELLE DANCHIN}

docteur en histoire contemporaine et en histoire, art et archéologie, chercheur partenaire à l'IRICE, UMR 8138 danchin.emmanuelle@gmail.com 